\title{
MULTIPLICITY OF THE SPECIAL FIBER OF BLOWUPS
}

\author{
ALBERTO CORSO, CLAUDIA POLINI, AND WOLMER V. VASCONCELOS
}

\begin{abstract}
Let $(R, \mathfrak{m})$ be a Noetherian local ring and let $I$ be an $\mathfrak{m}$-primary ideal. In this paper we give sharp bounds on the multiplicity of the special fiber ring $\mathcal{F}$ of $I$ in terms of other well-known invariants of $I$. A special attention is then paid in studying when equality holds in these bounds, with a particular interest in the unmixedness or, better, the Cohen-Macaulayness of $\mathcal{F}$.
\end{abstract}

\section{INTRODUCTION}

Let $(R, \mathfrak{m})$ be a Noetherian local ring with dimension $d>0$ and infinite residue field and let $I$ be an $R$-ideal. Three algebras, collectively referred to as blowup algebras of $I$, play an important role in the process of blowing up the variety $\operatorname{Spec}(R)$ along the subvariety $V(I)$ : namely, the Rees algebra $\mathcal{R}(=\mathcal{R}(I)=R[I t])$, the associated graded ring $\mathcal{G}(=\mathcal{G}(I))$, and the special fiber ring $\mathcal{F}(=\mathcal{F}(I))$ of $I$

$$
\mathcal{R}=\bigoplus_{m=0}^{\infty} I^{m} t^{m}, \quad \mathcal{G}=\mathcal{R} / I \mathcal{R}, \quad \mathcal{F}=\mathcal{R} / \mathfrak{m} \mathcal{R} .
$$

The blowup algebras are also extensively used as the means to examine diverse properties of the ideal $I$. Therefore, much attention has been paid in the past to find under which circumstances these objects have a good structure. In this article we focus on the special fiber ring $\mathcal{F}$, as there is lack of knowledge about its properties in comparison to the other two blowup algebras of $I$. From an algebraic point of view, $\mathcal{F}$ yields (asymptotic) information about the ideal $I$. For example, its Hilbert function is the numerical function that measures the growth of the minimal number of generators $\mu\left(I^{m}\right)$ of the powers of $I$. For $m \gg 0$ this function is a polynomial in $m$ of degree $\operatorname{dim} \mathcal{F}-1$, whose leading coefficient $f_{0}=f_{0}(I)$ is called the multiplicity (or degree) of the special fiber ring $\mathcal{F}$. Another important data attached to $\mathcal{F}$ is its (Krull) dimension, dubbed the analytic spread $\ell$ of $I$. It is bounded below by the height $g$ of $I$ and bounded above by the dimension of $R$. It coincides with the minimal number of generators of any minimal reduction of $I$. A minimal reduction - a notion that has been crucial in the study of the Rees algebra of an ideal, as it carries most of the information about the original ideal but, in general, with fewer generators - arise from a Noether normalization of $\mathcal{F}$.

AMS 2000 Mathematics Subject Classification. Primary 13A30, 13B21, 13D40; Secondary 13H10, 13 H15.

Part of this work was done while the authors were members of the Mathematical Sciences Research Institute (Berkeley) during the Fall 2002. They would like to thank that institution as well as the organizers of the year long program in Commutative Algebra for the stimulating atmosphere that this event provided. The last two authors also gratefully acknowledge partial support from the NSF. 
From a more geometric perspective, $\operatorname{Proj}(\mathcal{F})$ corresponds to the fiber over the closed point of the blowup of $\operatorname{Spec}(R)$ along $V(I)$. When $R$ is a standard graded domain over a field $k$ and $I$ is the $R$-ideal generated by forms $a_{0}, \ldots, a_{n}$ of the same degree, then $\mathcal{F}$ describes the homogeneous coordinate ring of the image of the rational map $\operatorname{Proj}(R) \rightarrow \mathbb{P}_{k}^{n}$ given by $\left(a_{0}, \ldots, a_{n}\right)$. As a special case this construction yields homogeneous coordinate rings of Gauss images and of secant varieties. More recently, special fiber rings also find an application in the theory of evolutions, due to Mazur [14 and inspired by the work of Wiles [31] on semistable curves. Indeed, in this context, Hübl and Huneke [9] were the first to use the special fiber ring $\mathcal{F}$ in studying the issue of the integral closedness of the ideal $\mathfrak{m} I$. See 4 ] for subsequent work.

We now describe the content of the paper. In Section 2 we prove our main results, which deal with an m-primary ideal $I$ of a local Cohen-Macaulay ring $R$. In Theorem 2.1] we show that the multiplicity $f_{0}$ of the special fiber ring $\mathcal{F}$ satisfies the inequality

$$
f_{0} \leq e_{1}-e_{0}+\lambda(R / I)+\mu(I)-d+1
$$

where $e_{0}=e_{0}(I)$ and $e_{1}=e_{1}(I)$ are the first two coefficients of the Hilbert polynomial of $I$ and $\lambda(\cdot)$ denotes the length function. We recall that for $m \gg 0$ the Hilbert-Samuel function of an $\mathfrak{m}$-primary ideal $I$ of a local Cohen-Macaulay ring $R$ - that is the numerical function that measures the growth of $\lambda\left(R / I^{m}\right)$ for all $m \geq 1$ - is a polynomial in $m$ of degree $d$, whose coefficients $e_{0}, e_{1}, \ldots, e_{d}$ are called the Hilbert coefficients of $I$. The leading term $e_{0}$ of this polynomial is called the multiplicity (of the associated graded ring) of $I$ : It is the only coefficient which is geometrically well understood as it equals $\lambda(R / J)$, for any minimal reduction $J$ of $I$.

Even though equality in the above bound does not assure the Cohen-Macaulayness of $\mathcal{F}$ (see Example 2.3), nevertheless it yields useful information about the associated primes of $\mathcal{F}$. In fact, in Theorem 2.5 we show that if $f_{0}=e_{1}-e_{0}+\lambda(R / I)+\mu(I)-d+1$, then $\mathcal{F}$ is unmixed. In particular the ideals $\mathfrak{m} I^{m}$ are integrally closed for all $m$, whenever $\mathcal{R}$ is normal (see Corollary [2.8). An immediate consequence of Theorem [2.5] is that if $\operatorname{depth} \mathcal{G} \geq d-1$ then $\mathcal{F}$ is Cohen-Macaulay (see Corollary 2.61).

Another motivation in finding bounds on $f_{0}$ in terms of natural data attached to the ideal $I$ is based on the general philosophy that a good control on the multiplicity $f_{0}$ coupled with depth information on $\mathcal{F}$ allow to bound the reduction number $r$ of the ideal. We recall that $J$ is a reduction of $I$ if $I^{r+1}=J I^{r}$ for some non-negative integer $r$ or, equivalently, if the inclusion of Rees algebras $\mathcal{R}(J) \hookrightarrow \mathcal{R}(I)$ is module finite [15. The least such $r$ is called the reduction number $r_{J}(I)$ of $I$ with respect to $J$. One then defines the reduction number of $I$ to be the least $r_{J}(I)$, where $J$ varies over all minimal reductions of $I$. A reduction is said to be minimal if it is minimal with respect to containment. In other words, the reduction number of an ideal is a key control element of the blowup algebras, often measuring the interplay among the other invariants of the ideal.

For example, if $I$ is generated by forms of the same degree then $\mathcal{F}$ is a domain and the reduction number $r$ of $I$ is bounded by $f_{0}$. Thus, in Section 3 we use the bounds 
on $f_{0}$ established earlier in the paper to obtain bounds on the reduction number of an $\mathfrak{m}$-primary ideal $I$. For instance, if the residue field $R / \mathfrak{m}$ has characteristic 0 and $f_{0}=$ $e_{1}-e_{0}+\lambda(R / I)+\mu(I)-d+1$ we conclude that $r \leq e_{1}-e_{0}+\lambda(R / I)+\mu(I)-d$ (see Corollary 3.1). If, in addition, $\mathcal{F}$ is Cohen-Macaulay this bound can be improved and in Corollary 3.5] we show that $r \leq e_{1}-e_{0}+\lambda(R / I)+1$. This latter bound has been shown without any additional assumption by Rossi [20] if the dimension of the ring is at most 2 . It seems to hold in full generality.

In Section 4 we strengthen the bound on $f_{0}$ obtained in Section 1 . With the same assumption as in Theorem 2.1 we first prove that the multiplicity $f_{0}$ of the special fiber ring $\mathcal{F}$ of $I$ satisfies the tighter bound $f_{0} \leq e_{1}-e_{0}+\lambda(R / \widetilde{I})+\mu(\widetilde{I})-d+1$, where $\widetilde{I}$ denotes the Ratliff-Rush closure of $I$ (see Corollary 4.2). We recall that if $I$ is an m-primary ideal containing a regular element, then the Ratliff-Rush closure $\widetilde{I}$ of an ideal $I$ is the largest ideal containing $I$ with the same Hilbert polynomial as $I$ [19]. We can actually improve the estimate on $f_{0}$ by giving a different derivation, that we carry out in Theorem 4.1, of the construction, originally due to Shah [22], of a canonical sequence of ideals containing $I$ with partially identical Hilbert polynomials to the one of $I$. In Corollary 4.3 we then show that $f_{0} \leq e_{1}-e_{0}+\lambda(R / \check{I})+\mu(\check{I})-d+1$, where $\check{I}$ is the degree one component of the $S_{2}$-ification of the Rees algebra of $I$. The ideal $\check{I}$ can also be characterized as the largest ideal containing $I$ with the same $e_{0}$ and $e_{1}$ as $I[\mathbf{2}$. The crucial issue is then to show that the multiplicity $f_{0}$ of the special fiber ring of $I$ is unchanged when passing to the special fiber ring of $\check{I}$.

The main idea, behind the proofs of Theorem 2.1 and Theorem 2.5 is the use of the Sally module $S_{J}(I)$ of $I$ with respect to a minimal reduction $J$, which is defined by the following exact sequence of $\mathcal{R}(J)$-modules

$$
0 \rightarrow I \mathcal{R}(J) \longrightarrow I \mathcal{R}(I) \longrightarrow S_{J}(I)=\bigoplus_{m \geq 2} I^{m} / I J^{m-1} \rightarrow 0
$$

introduced by Vasconcelos 24. In this paper, though, we make use of a new approach to $S_{J}(I)$ by means of the following exact sequence

$$
\mathcal{R}(J) \oplus \mathcal{R}(J)^{n-\ell}[-1] \longrightarrow \mathcal{R}(I) \longrightarrow S_{J}(I)[-1] \rightarrow 0,
$$

where $n=\mu(I)$ and $\ell$ is the analytic spread of $I$. The advantage of this approach is that it is suitable to study the multiplicity of the special fiber ring $\mathcal{F}$ of any ideal, not necessarily $\mathfrak{m}$-primary. In addition, this approach avoids the customary technique in the theory of Hilbert function of going modulo a superficial sequence.

\section{General Bounds}

We start describing some general bounds on the multiplicity $f_{0}$ of the special fiber ring $\mathcal{F}$ of $I$. A special attention is then paid in studying when equality holds in these bounds, with a particular interest in the unmixedness or, better, the Cohen-Macaulayness of $\mathcal{F}$. 
2.1. Upper bounds. In [28, 2.4] Vasconcelos has shown that the multiplicity $f_{0}$ of the special fiber ring $\mathcal{F}$ of an $\mathfrak{m}$-primary ideal $I$ of a local Cohen-Macaulay ring $R$ satisfies the inequality

$$
f_{0} \leq \min \left\{e_{0}, e_{1}+1\right\}
$$

where $e_{0}$ and $e_{1}$ are the first two coefficients of the Hilbert polynomial of $I$. In the following theorem we give a better bound on $f_{0}$ of a different nature, using the structure of the Sally module $S_{J}(I)$ of $I$ with respect to a minimal reduction $J$.

Theorem 2.1. Let $(R, \mathfrak{m})$ be a local Cohen-Macaulay ring of dimension $d>0$ and infinite residue field. Let $I$ be an $\mathfrak{m}$-primary ideal. Then the multiplicity $f_{0}$ of the special fiber ring $\mathcal{F}$ of I satisfies

$$
f_{0} \leq e_{1}-e_{0}+\lambda(R / I)+\mu(I)-d+1 .
$$

Proof. Let $J$ be a minimal reduction of $I$ and write $I=\left(J, a_{1}, \ldots, a_{n-d}\right)$, where $n=\mu(I)$ and $d=\mu(J)=\ell$. We now consider the Sally module $S_{J}(I)$ of $I$ with respect to $J$ [24 2.1]. However, we find it more suitable for our purposes to approach $S_{J}(I)$ by means of a new exact sequence, namely

$$
\mathcal{R}(J) \oplus \mathcal{R}(J)^{n-d}[-1] \stackrel{\varphi}{\longrightarrow} \mathcal{R}(I) \longrightarrow S_{J}(I)[-1] \rightarrow 0,
$$

where $\varphi$ is the map defined by $\varphi\left(r_{0}, r_{1}, \ldots, r_{n-d}\right)=r_{0}+r_{1} a_{1} t+\ldots+r_{n-d} a_{n-d} t$, for any element $\left(r_{0}, r_{1}, \ldots, r_{n-d}\right) \in \mathcal{R}(J) \oplus \mathcal{R}(J)^{n-d}[-1]$. Tensoring the above exact sequence with $R / \mathfrak{m}$ yields

$$
\mathcal{F}(J) \oplus \mathcal{F}(J)^{n-d}[-1] \longrightarrow \mathcal{F}(I) \longrightarrow S_{J}(I)[-1] \otimes R / \mathfrak{m} \rightarrow 0 .
$$

As the three modules in (21) have the same dimension, we obtain the multiplicity estimate

$$
f_{0} \leq \operatorname{deg}\left(S_{J}(I)[-1] \otimes R / \mathfrak{m}\right)+\operatorname{deg}\left(\mathcal{F}(J) \oplus \mathcal{F}(J)^{n-d}[-1]\right) .
$$

Since $S_{J}(I) \otimes R / \mathfrak{m}$ is a homomorphic image of $S_{J}(I)$, its multiplicity is bounded by the one of $S_{J}(I)$, which - according to [24, 3.3] - is $e_{1}-e_{0}+\lambda(R / I)$. On the other hand, $\mathcal{F}(J) \oplus \mathcal{F}(J)^{n-d}[-1]$ is a free $\mathcal{F}(J)$-module of rank $n-d+1$. Thus, its multiplicity is $n-d+1$, since $\mathcal{F}(J)$ is isomorphic to a ring of polynomials.

Proposition 2.2. Let $(R, \mathfrak{m})$ be a local Cohen-Macaulay ring of dimension $d>0$ and infinite residue field. Let $I$ be an $\mathfrak{m}$-primary ideal. We have that

$$
f_{0} \leq e_{1}-e_{0}+\lambda(R / I)+\mu(I)-d+1 \leq e_{1}+1 .
$$

In particular, if $f_{0}=e_{1}+1$ then $I$ has minimal multiplicity in the sense of S. Goto, that is $\mathfrak{m} I=\mathfrak{m} J$ for any minimal reduction $J$ of $I$. If, in addition, $R$ is a local Gorenstein ring then $\mathcal{F}$ is Cohen-Macaulay.

Proof. Without loss of generality, we may assume $I \neq J$. As $e_{0}=\lambda(R / J)$, from the identity $\lambda(I / \mathfrak{m} I)+\lambda(\mathfrak{m} I / \mathfrak{m} J)=\lambda(I / J)+\lambda(J / \mathfrak{m} J)$ it readily follows that $-e_{0}+\lambda(R / I)+$ $\mu(I)-d=-\lambda(\mathfrak{m} I / \mathfrak{m} J)$. Thus

$$
f_{0} \leq e_{1}-e_{0}+\lambda(R / I)+\mu(I)-d+1=e_{1}-\lambda(\mathfrak{m} I / \mathfrak{m} J)+1 \leq e_{1}+1,
$$


as desired. Therefore, the equality $f_{0}=e_{1}+1$ forces $\mathfrak{m} I=\mathfrak{m} J$ and hence $\mathfrak{m}=J: I$. If, in addition, $R$ is a local Gorenstein ring, we have that $I=J: \mathfrak{m}$ as well. Thus $I^{2}=J I$ by 3 2.2]. Finally, the Cohen-Macaulayness of $\mathcal{F}$ follows from [12,3.3].

We notice that equality in (11) - and a fortiori in the bound established in Theorem 2.1] - does not imply, in general, the Cohen-Macaulayness of $\mathcal{F}$ if the $\operatorname{ring} R$ is not Gorenstein.

Example 2.3. Given a field $k$, let $R$ be the power series ring $k \llbracket T_{1}, T_{2}, T_{3}, T_{4}, T_{5} \rrbracket$ modulo the ideal generated by the two by two minors of the matrix

$$
\varphi=\left[\begin{array}{llll}
T_{1} & T_{2} & T_{3} & T_{4} \\
T_{2} & T_{3} & T_{4} & T_{5}
\end{array}\right]
$$

The ring $R$ is a local Cohen-Macaulay ring of dimension two and type three. Let $t_{i}$ denote the image of $T_{i}$ in $R$ and consider the ideal $I=\left(t_{1}, t_{2}, t_{4}, t_{5}\right)$. Then

$$
f_{0}=4=e_{0}=e_{1}+1=e_{1}-e_{0}+\lambda(R / I)+\mu(I)-d+1 .
$$

However, the special fiber ring $\mathcal{F}$ of $I$ has depth one.

Next, we give a very general estimate for the multiplicity of $\mathcal{F}$, which is valid for any ideal $I$ of a Noetherian local ring $(R, \mathfrak{m})$ with infinite residue field. We observe that a characterization of the Cohen-Macaulayness of $\mathcal{F}$ in terms of the degree of $\mathcal{F}$ can also be found in [23, 5].

Proposition 2.4. Let $(R, \mathfrak{m})$ be a Noetherian local ring with infinite residue field and let I be an ideal with a minimal reduction $J$. If $r=r_{J}(I)$ then

$$
f_{0} \leq 1+\sum_{j=1}^{r} \mu\left(I^{j} / J I^{j-1}\right)
$$

and equality holds if and only if $\mathcal{F}$ is Cohen-Macaulay.

Proof. Set $A=\mathcal{F}(J)=k\left[x_{1}, \ldots, x_{\ell}\right]$, where $\ell$ is the analytic spread of $I$, and $\mathcal{F}(I)=$ $\bigoplus F_{j}$. Let $N$ be the minimal number of generators of $\mathcal{F}(I)$ as a $A$-module. As $f_{0}=$ $j \geq 0$

$\operatorname{deg} \mathcal{F}(I) \leq N \operatorname{deg} A=N$, we only need to estimate $N$. As an $A$-module, $\mathcal{F}(I)$ is minimally generated by

$$
\operatorname{dim}_{k}\left(\mathcal{F}(I) / A_{+} \mathcal{F}(I)\right)=1+\sum_{j=1}^{r} \operatorname{dim}_{k}\left(F_{j} /\left(x_{1}, \ldots, x_{\ell}\right) F_{j-1}\right)
$$

elements. But these summands are $\mu\left(I^{j} / J I^{j-1}\right)$. The second assertion follows from the standard criterion for Cohen-Macaulayness as applied to graded $A$-modules.

Note that the ideal $I$ of Example 2.3 has reduction number 2 with respect to the ideal $J=\left(t_{1}, t_{2}\right)$. Moreover, $\mu(I / J)=2$ and $\mu\left(I^{2} / J I\right)=2$. Thus, the failure of the CohenMacaulayness of $\mathcal{F}$ is explained by the strict inequality $f_{0}=4<5=1+\mu(I / J)+\mu\left(I^{2} / J I\right)$. 
Even though the equality in the bound described in Theorem 2.1 does not assure the Cohen-Macaulayness of $\mathcal{F}$, nevertheless it yields useful information about the associated primes of $\mathcal{F}$.

Theorem 2.5. Let $(R, \mathfrak{m})$ be a Cohen-Macaulay local ring of dimension $d>0$ and infinite residue field. Let $I$ be an $\mathfrak{m}$-primary ideal. If $f_{0}=e_{1}-e_{0}+\lambda(R / I)+\mu(I)-d+1$, then $\mathcal{F}$ is unmixed.

Proof. We may assume that the reduction number of $I$ is strictly greater than 1 . From the sequence (2) in the proof of Theorem [2.1, we obtain the following diagram

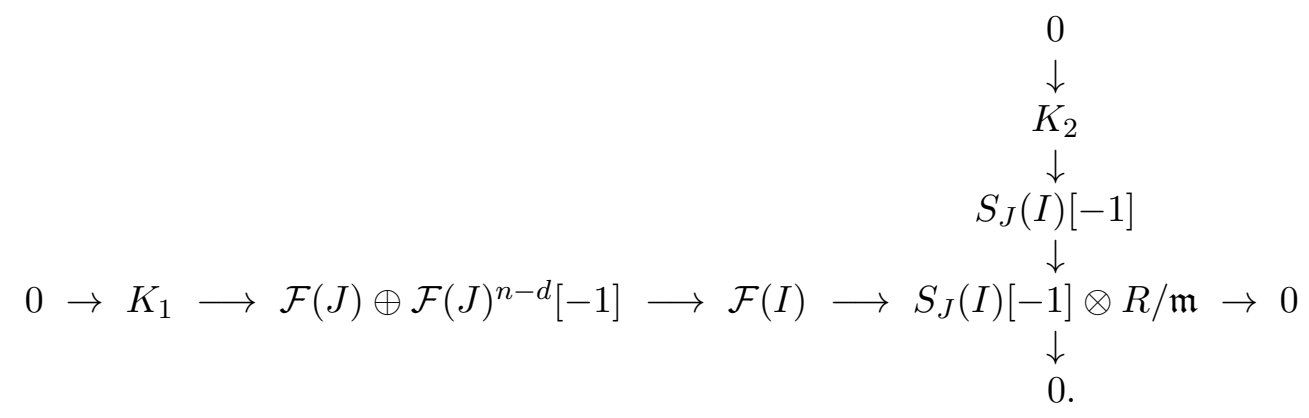

Now, the asserted equality $f_{0}=e_{1}-e_{0}+\lambda(R / I)+n-d+1$ implies that both $K_{1}$ and $K_{2}$ have multiplicity (degree) zero. But $K_{1}$ is a submodule of the free $\mathcal{F}(J)$-module $\mathcal{F}(J) \oplus$ $\mathcal{F}(J)^{n-d}[-1]$, so it is zero. Whereas any non-zero submodule of $S_{J}(I)$ must have positive multiplicity, since $S_{J}(I)$ has no associated primes of height $\geq 2$ (see [24, 2.2]). Thus $K_{2}$ is zero as well. Hence we have an exact sequence

$$
0 \rightarrow \mathcal{F}(J) \oplus \mathcal{F}(J)^{n-d}[-1] \longrightarrow \mathcal{F}(I) \longrightarrow S_{J}(I)[-1] \rightarrow 0,
$$

from which the desired conclusion follows.

Next, we observe that the previous theorem gives us a relation between the depths of $\mathcal{F}$ and $S_{J}(I)$, and a fortiori a relation between the depths of $\mathcal{F}$ and $\mathcal{G}$. In particular, good depth conditions on $\mathcal{G}$ and the equality in the bound established in Theorem 2.1 force the Cohen-Macaulayness of $\mathcal{F}$.

Corollary 2.6. Let $(R, \mathfrak{m})$ be a Cohen-Macaulay local ring of dimension $d>0$ and infinite residue field. Let $I$ be an $\mathfrak{m}$-primary ideal. If $f_{0}=e_{1}-e_{0}+\lambda(R / I)+\mu(I)-d+1$, then

$$
\operatorname{depth} \mathcal{F} \geq \min \{\operatorname{depth} \mathcal{G}+1, d\} .
$$

If, in addition, depth $\mathcal{G} \geq d-1$ then $\mathcal{F}$ is Cohen-Macaulay.

Proof. The inequality follows from a depth count on (3) and the equality $\operatorname{depth} S_{J}(I)=$ $\min \{\operatorname{depth} \mathcal{G}+1, d\}$, which follows from [30, 1.2.11] and [29, 2.1].

The associated primes of $\mathcal{F}$ play a role in the integral closedness of the product $\mathfrak{m} I$. To see how this comes about, we recall an observation of [9, 1.5], later refined in [4, 4.1]. Then, in Corollary 2.8, we give an application in its spirit. 
Proposition 2.7. Let $(R, \mathfrak{m})$ be a normal local domain of dimension $d>0$ with infinite residue field and suppose $I$ is a normal ideal of analytic spread $d$. If $\mathcal{F}$ is unmixed then $\overline{\mathfrak{m} I^{m}}=\mathfrak{m} I^{m}$ for all $m$.

Corollary 2.8. Let $(R, \mathfrak{m})$ be a normal Cohen-Macaulay local domain of dimension $d>0$ and infinite residue field. Let $I$ be an $\mathfrak{m}$-primary normal ideal. If $f_{0}=e_{1}-e_{0}+\lambda(R / I)+$ $\mu(I)-d+1$, then $\overline{\mathfrak{m} I^{m}}=\mathfrak{m} I^{m}$ for all $m$.

In the same fashion as Theorem 2.1] and Theorem 2.5] one can show a bound on the first two Hilbert coefficients of $I$, which possibly yields information on the associated graded ring $\mathcal{G}$ of $I$. The following inequality is of interest in the case of exotic local Cohen-Macaulay rings of small dimension, that are not regular local rings. The bound was proved by Elias and Valla in the case of the maximal ideal [7, Section 2].

Remark 2.9. Let $(R, \mathfrak{m})$ be a local Cohen-Macaulay ring of dimension $d>0$ and infinite residue field. Let $I$ be an $\mathfrak{m}$-primary ideal. Then the coefficients $e_{0}$ and $e_{1}$ of the Hilbert polynomial of $I$ satisfy

$$
2 e_{0}-e_{1} \leq \lambda(R / I)(\mu(I)-d+2) .
$$

Moreover, if equality holds one has that the associated graded ring $\mathcal{G}$ is unmixed; if, in addition, the Sally module $S_{J}(I)$ of $I$ with respect to a minimal reduction $J$ is CohenMacaulay then $\mathcal{G}$ is Cohen-Macaulay as well.

Another interesting example, where we can argue the Cohen-Macaulayness of $\mathcal{F}$ and hence the integral closedness of the ideals $\mathfrak{m} I^{m}$ for all $m$, is due to Huckaba and Huneke [10, 3.11]. It is the first explicit example of the failure in dimension three of a two-dimensional version (due to Sancho de Salas) of a vanishing theorem of Grauert and Riemenschneider. This example complements nicely a family of such examples over $\mathbb{C}$ constructed by Cutkosky $\mathbf{5}$, Section III].

Example 2.10. Let $k$ be a field of characteristic $\neq 3$ and set $R=k \llbracket x, y, z \rrbracket$, where $x, y, z$ are indeterminates. Let $P=\left(x^{4}, x\left(y^{3}+z^{3}\right), y\left(y^{3}+z^{3}\right), z\left(y^{3}+z^{3}\right)\right)$ and set $I=P+\mathfrak{m}^{5}$, where $\mathfrak{m}$ is the maximal ideal of $R$. The ideal $I$ is a 16 generated, normal, $\mathfrak{m}$-primary ideal with $\operatorname{depth} \mathcal{G}\left(I^{m}\right)=\operatorname{dim} R-1(=2)$ for all $m \geq 1$. Moreover, we have that $I^{4}=J I^{3}$, where $J=\left(x^{4}, z\left(y^{3}+z^{3}\right), y\left(y^{3}+z^{3}\right)+z^{5}\right)$. The Cohen-Macaulayness of $\mathcal{F}$ follows from Proposition 2.4 since we have that $f_{0}=16, \mu(I / J)=13$ and $\mu\left(I^{2} / J I\right)=\mu\left(I^{3} / J I^{2}\right)=1$. On the other hand, as far as the bound in Theorem 2.1] is concerned, we have that $f_{0}=$ $16<17=e_{1}-e_{0}+\lambda(R / I)+\mu(I)-d+1$. In fact one has that $e_{0}=76, e_{1}=48$ and $\lambda(R / I)=31$.

2.2. Lower bounds. Let $(R, \mathfrak{m})$ be a Noetherian local ring and let $I$ be an $\mathfrak{m}$-primary ideal. Let $R / I=A_{0} \supset A_{1} \supset \cdots \supset A_{s}=0$, with $\lambda\left(A_{i} / A_{i+1}\right)=1$ for $0 \leq i \leq s-1$ and $s=\lambda(R / I)$, be a composition series of $R / I$. Thus we can find $u_{i} \in R$ such that $A_{i}=\left(A_{i+1}, u_{i}\right)$ and $\mathfrak{m} u_{i} \in A_{i+1}$. On the other hand, this composition series induces a 
filtration $\mathcal{G}=A_{0} \mathcal{G} \supset A_{1} \mathcal{G} \supset \cdots \supset A_{s} \mathcal{G}=0$ on the associated graded ring $\mathcal{G}$ of $I$, whose factors $A_{i} \mathcal{G} / A_{i+1} \mathcal{G} \cong u_{i} \mathcal{G}$ are $\mathcal{F}(I)$-modules. A bookkeeping in the family of epimorphisms $\mathcal{F} \longrightarrow u_{i} \mathcal{G} \rightarrow 0$ yields the bound $e_{0} \leq \lambda(R / I) f_{0}$, or, equivalently,

$$
\frac{e_{0}}{\lambda(R / I)} \leq f_{0}
$$

It has been proved in $[\mathbf{8}, 2.6]$ that if the associated primes of $\mathcal{F}$ have the same dimension then equality in the above formula is equivalent to $I^{m} / I^{m+1}$ being $R / I$-free for all $m \geq 1$. In this situation, $R$ is said to be normally flat along $I$. We also observe that an earlier version of this latter characterization can be found in [23, 8].

\section{SPECIAL BOUndS}

In [21 2.2], Sally proved that the reduction number of the maximal ideal of a local CohenMacaulay ring of dimension $d$ is bounded by $d ! e_{0}-1$. In cite [26, 6.12 and 6.16], Vasconcelos improved this bound and for any m-primary ideal he showed that $r \leq d\left(e_{0}-2\right)+1$. In this Section we give bounds on the reduction number of an $\mathfrak{m}$-primary ideal via the estimates on the multiplicity of the special fiber ring $\mathcal{F}$ obtained earlier.

Corollary 3.1. Let $(R, \mathfrak{m})$ be a Cohen-Macaulay local ring of dimension $d>0$. Let $I$ be an $\mathfrak{m}$-primary ideal with reduction number $r$. If the residue field $R / \mathfrak{m}$ has characteristic 0 and $f_{0}=e_{1}-e_{0}+\lambda(R / I)+\mu(I)-d+1$, then

$$
r \leq e_{1}-e_{0}+\lambda(R / I)+\mu(I)-d .
$$

Proof. From Theorem 2.5 we conclude that $\mathcal{F}$ is unmixed. Hence the reduction number of $I$ is at most $f_{0}-1$, by a result of Vasconcelos [25, 7 and 9] (see also [27, 2.2(a)]).

We note that the above bound on $r$ can actually be sharpened if the special fiber ring $\mathcal{F}$ is Cohen-Macaulay: See Corollary 3.5 below.

Remark 3.2. Let $R$ be a local Cohen-Macaulay ring of dimension $d>0$ and infinite residue field. Let $I$ be an $\mathfrak{m}$-primary ideal of reduction number $r$. If the special fiber $\operatorname{ring} \mathcal{F}$ of $I$ is Cohen-Macaulay then

$$
r \leq f_{0}-\mu(I)+d
$$

where $f_{0}$ is the multiplicity of $\mathcal{F}$.

Proof. We seek to estimate the degree $r$ of the last basis element of the vector space $\mathcal{V}=\mathcal{F} /(\mathbf{z}) \mathcal{F}$, where $\mathbf{z}=z_{1}, \ldots, z_{d}$ is a superficial sequence. As $\mathcal{V}$ has one basis element in degree zero, $\mu(I)-d$ basis elements in degree one and at least one basis element in each degree, we get $1+\mu(I)-d+r-1 \leq \operatorname{dim} \mathcal{V}=f_{0}$. Hence our claim.

The assumption on the Cohen-Macaulayness of $\mathcal{F}$ is necessary as the next example shows.

Example 3.3. Let $R=k \llbracket t^{6}, t^{11}, t^{15}, t^{31} \rrbracket$, where $k$ is an infinite field, and let $I=\left(t^{6}, t^{11}, t^{31}\right)$. The ideal $I$ has height 1 , analytic spread 1 and reduction number 2. Furthermore $\mathcal{G}(I)$ is Cohen-Macaulay, but $\mathcal{F}$ is not Cohen-Macaulay. One has that $e_{0}=6, e_{1}=5$ and $f_{0}=3$. Thus $r=2>1=f_{0}-\mu(I)+d$. On the other hand one has $r=2=e_{1}-e_{0}+\lambda(R / I)+1$. 
Next, we point out a consequence of Remark 3.2 and Theorem 2.1 in the special case of ideals with reduction number one. It easily recovers an earlier result of Shah [23, $7(b)]$.

Corollary 3.4. Let $R$ be a local Cohen-Macaulay ring of dimension $d>0$ and infinite residue field. Let $I$ be an $\mathfrak{m}$-primary ideal of reduction number one. Then the multiplicity $f_{0}$ of the special fiber ring $\mathcal{F}$ of $I$ is given by $f_{0}=\mu(I)-d+1$.

Proof. As $I$ has reduction number one it follows from [11, 2.1] and [16, 3.2 and 3.3] that $e_{1}-e_{0}+\lambda(R / I)=0$. Thus, Theorem 2.1 gives that $f_{0} \leq \mu(I)-d+1$. On the other hand, the special fiber ring of $I$ is Cohen-Macaulay by [12,3.3], so that the reverse inequality follows from Abhyankar's bound [1, 1].

The following result has been proved without any assumption on the special fiber ring by Rossi for local Cohen-Macaulay rings of dimension at most two [20, 1.5]. It also holds whenever $\operatorname{depth} \mathcal{G} \geq \operatorname{dim} R-1$.

Corollary 3.5. Let $R$ be a local Cohen-Macaulay ring of dimension $d>0$ and infinite residue field. Let $I$ be an $\mathfrak{m}$-primary ideal of reduction number $r$. If the special fiber ring $\mathcal{F}$ of $I$ is Cohen-Macaulay then

$$
r \leq e_{1}-e_{0}+\lambda(R / I)+1 .
$$

Proof. By combining Remark 3.2 and Theorem 2.1 one has that

$$
\begin{aligned}
r & \leq f_{0}-\mu(I)+d & & \text { (by Remark [3.2) } \\
& \leq\left(e_{1}-e_{0}+\lambda(R / I)+\mu(I)-d+1\right)-\mu(I)+d & & \text { (by Theorem 2.1) } \\
& =e_{1}-e_{0}+\lambda(R / I)+1, & &
\end{aligned}
$$

as claimed.

If $R$ is a 2-dimensional regular local ring, we obtain an estimate on the reduction number $r$ and on the multiplicity $f_{0}$ of an ideal $I$ which does not explicitly involve the Hilbert coefficients of the ideal $I$.

Corollary 3.6. Let $(R, \mathfrak{m})$ be a 2-dimensional regular local ring of infinite residue field. Let $I$ be an $\mathfrak{m}$-primary ideal of reduction number $r$. Then

$$
r \leq \lambda(\bar{I} / I)+1 \quad \text { and } \quad f_{0} \leq \lambda(\bar{I} / I)+\mu(I)-1,
$$

where $\bar{I}$ denotes the integral closure of $I$.

Proof. We have that $e_{1}(I) \leq \bar{e}_{1}(I)$ by [17], where $\bar{e}_{1}(I)$ is the corresponding Hilbert coefficient arising from the filtration given by the integral closure of the powers of $I$ rather than the $I$-adic filtration. On the other hand, using the fact $\bar{I}$ is a normal ideal of reduction number one [13,5.5], one has that $\bar{e}_{1}(I)=e_{1}(\bar{I})=\lambda(\bar{I} / J)$ for any minimal reduction $J$ of I. As $e_{0}=\lambda(R / J)$, one finally concludes from Rossi's bound [20, 1.5] that

$$
r \leq e_{1}-e_{0}+\lambda(R / I)+1 \leq \lambda(\bar{I} / J)-e_{0}+\lambda(R / I)+1=\lambda(\bar{I} / I)+1,
$$

as claimed. 
Remark 3.7. Observe that whenever $\bar{I} \neq \mathfrak{m}$ one has that the above bounds translate into

$$
r \leq e_{0}-2 \quad \text { and } \quad f_{0} \leq \lambda(R / I)+\mu(I)-3 .
$$

The first one compares favorably with Vasconcelos' earlier bound, which says $r \leq 2 e_{0}-3$.

\section{Partially identical Hilbert polynomials}

To compare $I$ to its integral closure $\bar{I}$ it is useful to track their numerical measures as expressed by appropriate Hilbert functions. An approach was given in [22, 1] to construct a 'canonical' sequence of ideals $I \subset I_{0} \subset I_{1} \subset \cdots \subset I_{d-1} \subset I_{d}=\bar{I}$, with Hilbert polynomials partially identical to the one of $I$. More recently, Ciuperca [2, 2.5] has instead shown a connection with the $S_{2}$-ification of the Rees algebra of $I$.

We give below another derivation of Shah's result as it will be relevant for an improvement of our earlier bounds on $f_{0}$.

Theorem 4.1. Let $(R, \mathfrak{m})$ be an analytically equidimensional local domain of dimension $d$ and let $I$ be an $\mathfrak{m}$-primary ideal. There exists a unique, largest ideal $I \subset I_{j} \subseteq \bar{I}$, with $0 \leq$ $j \leq d$ and the property that the corresponding Hilbert polynomial has coefficients satisfying

$$
e_{i}\left(I_{j}\right)=e_{i}(I), \quad i=0, \ldots, d-j .
$$

Proof. Let $A$ be the extended Rees algebra $R\left[I t, t^{-1}\right]$ of $I$. For each integer $1 \leq j \leq d+1$, let $B^{(j)}$ be the subring of $C=R\left[t, t^{-1}\right] \cap \overline{R\left[I t, t^{-1}\right]}$ defined by $B^{(j)}=\left\{h \in C \mid \operatorname{ht}\left(A:_{A} h\right) \geq j\right\}$. This gives a filtration of subalgebras $A=B^{(d+2)} \subset B^{(d+1)} \subset \cdots \subset B^{(2)} \subset B^{(1)}$. By considering only the component $A^{(j)}$ of $B^{(j)}$ in non-negative degrees (since they all have the same components in negative degrees) we have

$$
\begin{aligned}
A^{(1)} & =\text { integral closure of } R[I t] \text { in } R[t] \\
A^{(2)} & =S_{2} \text {-ification of } R[I t] \\
A^{(d+1)} & =\text { Ratliff-Rush closure of } R[I t] \\
A^{(d+2)} & =\text { Rees algebra } R[I t] \text { of } I .
\end{aligned}
$$

We are now ready to relate these algebras to the ideals $I_{j}$ and to prove the assertion about their Hilbert polynomials. Set $I_{j}$ to be the ideal defined by the component of degree 1 of $A^{(d+1-j)}$. We have exact sequences of finitely generated modules over $R\left[I t, t^{-1}\right]$

$$
0 \rightarrow R\left[I t, t^{-1}\right] \longrightarrow R\left[I_{j} t, t^{-1}\right] \longrightarrow M_{j} \rightarrow 0,
$$

where $M_{j}$ is a module of dimension at most $d+1-(d+1-j)=j$.

Tensoring this sequence by $R\left[I t, t^{-1}\right] /\left(t^{-1}\right)$, we obtain two exact sequences of graded modules:

$$
\begin{gathered}
0 \rightarrow K_{j}[+1] \longrightarrow M_{j}[+1] \stackrel{t^{-1}}{\longrightarrow} M_{j} \longrightarrow C_{j} \rightarrow 0, \\
0 \rightarrow K_{j} \longrightarrow \mathcal{G}(I) \longrightarrow \mathcal{G}\left(I_{j}\right) \longrightarrow C_{j} \rightarrow 0,
\end{gathered}
$$

Note that (41) was induced by multiplication by $t^{-1}$, an endomorphism which is nilpotent on $M_{j}$; it follows that (see [6, 12.1]) the Krull dimension of all the modules in this sequence is 
the same. One can further assert that the multiplicities of $K_{j}$ and of $C_{j}$ match. As a consequence, adding in (5) the Hilbert polynomials, we obtain that the Hilbert polynomials of $\mathcal{G}(I)$ and of $\mathcal{G}\left(I_{j}\right)$ have matching coefficients not only down to degree $j$ (which is guaranteed by the codimension of $M_{j}$ ) but one position further down.

One can actually do slightly better in the estimate of $f_{0}$ established in Theorem 2.1]

Corollary 4.2. Let $R$ be a local Cohen-Macaulay ring of dimension $d$ and infinite residue field. Let $I$ be an $\mathfrak{m}$-primary ideal. Then the multiplicity $f_{0}$ of the special fiber ring $\mathcal{F}$ of $I$ satisfies

$$
f_{0} \leq e_{1}-e_{0}+\lambda(R / \widetilde{I})+\mu(\widetilde{I})-d+1,
$$

where $\widetilde{I}$ is the Ratliff-Rush closure of $I$.

Proof. The special fiber rings $\mathcal{F}(\widetilde{I})$ and $\mathcal{F}(I)$ have the same multiplicity, since these two algebras differ at most in a finite number of components. Now observe that the relations

$$
\lambda(R / \widetilde{I})=\lambda(R / I)-\lambda(\widetilde{I} / I) \quad \text { and } \quad \mu(\widetilde{I}) \leq \mu(I)+\lambda(\widetilde{I} / I)
$$

implies that $\lambda(R / \widetilde{I})+\mu(\widetilde{I}) \leq \lambda(R / I)+\mu(I)$. Finally, since the Hilbert coefficients are the same for both ideals $\widetilde{I}$ and $I$, we conclude that the formula

$$
f_{0} \leq e_{1}-e_{0}+\lambda(R / \widetilde{I})+\mu(\widetilde{I})-d+1
$$

sharpens Theorem 2.1

An additional improvement occurs from the following consideration. Let $\check{I}$ denote the largest ideal containing $I$ such that in the embedding of Rees algebras

$$
0 \rightarrow \mathcal{R}(I) \longrightarrow \mathcal{R}(\check{I}) \longrightarrow C \rightarrow 0,
$$

$\operatorname{dim} C \leq d-1$. Thus, $\check{I}$ is the ideal $I_{d-1}$ in the terminology established in the proof of Theorem 4.1] that is the degree one component of the $S_{2}$-ification of the Rees algebra of $I$.

Corollary 4.3. Let $R$ be a local Cohen-Macaulay ring of dimension $d>0$ and infinite residue field. Let $I$ be an $\mathfrak{m}$-primary ideal. Then the multiplicity $f_{0}$ of the special fiber ring $\mathcal{F}$ of I satisfies

$$
f_{0} \leq e_{1}-e_{0}+\lambda(R / \check{I})+\mu(\check{I})-d+1 .
$$

Proof. According to Theorem $4.1 \check{I}$ is also the largest ideals containing $I$ with the same values for the Hilbert coefficients $e_{0}$ and $e_{1}$. From equation (7) it follows that there is an induced sequence

$$
0 \rightarrow D \longrightarrow \mathcal{F}(I) \longrightarrow \mathcal{F}(\check{I}) \longrightarrow C / \mathfrak{m} C \rightarrow 0
$$

of finitely generated $\mathcal{F}(I)$-modules such that the dimensions of $C / \mathfrak{m} C$ and $D$ are at most $d-1$. Indeed, in the case of $D$ one has the map $\operatorname{Tor}_{1}(C, R / \mathfrak{m}) \longrightarrow D \rightarrow 0$, which justifies our assertion about the dimension. Thus, the special fiber rings $\mathcal{F}(\check{I})$ and $\mathcal{F}(I)$ have the same multiplicity. Therefore if we write formula (6) for $\check{I}$ instead of $\widetilde{I}$,

$$
f_{0} \leq e_{1}-e_{0}+\lambda(R / \check{I})+\mu(\check{I})-d+1
$$

we can only achieve gains, as the function $\lambda(R / \cdot)+\mu(\cdot)$ is monotone non-increasing. 
Corollary 4.4. Let $R=k[x, y]$ with $k$ a field of characteristic zero and $x, y$ variables, and let $\mathfrak{m}$ denote the maximal homogeneous ideal of $R$. Let $I$ be an $\mathfrak{m}$-primary monomial ideal with a monomial 2-generated reduction. Then the multiplicity $f_{0}$ of the special fiber ring $\mathcal{F}$ of $I$ is given by $f_{0}=\mu(\check{I})-1$, where $\check{I}$ is the degree one component of the $S_{2}$-ification of the Rees algebra of $I$.

Proof. For any 2-generated reduction $J$ of $I$, it follows from 18 that $\check{I}=J:\left(J^{r}: I^{r}\right)$, where $r$ is the reduction number of $I$ with respect to $J$. Moreover, $\check{I}$ has reduction number one. Our assertion then follows from Corollary 3.4

The assertion of Corollary 4.4 seems to hold for all $\mathfrak{m}$-primary ideals of a two-dimensional regular local ring. The following example is taken from [2, 3.3], where it is erroneously stated that $\mathcal{R}(\check{I})$ is not Cohen-Macaulay.

Example 4.5. Let $R=k[x, y]_{(x, y)}$ with $k$ a field of characteristic zero. The ideal $I=$ $\left(x^{8}, x^{3} y^{2}, x^{2} y^{4}, y^{8}\right)$ has $S_{2}$-ification $\check{I}=\left(x^{8}, x^{3} y^{2}, x^{2} y^{4}, x y^{6}, y^{8}\right)$. We have that $f_{0}=4, e_{0}=$ 40 and $e_{1}=12$ whereas $\lambda(R / I)=30$ and $\lambda(R / \check{I})=28$. Thus in one case we have that $f_{0}=4<5=e_{1}-e_{0}+\lambda(R / I)+\mu(I)-d+1$ while in the other we have that $f_{0}=4=e_{1}-e_{0}+\lambda(R / \check{I})+\mu(\check{I})-d+1$. By choosing $J=\left(x^{8}+y^{8}+x^{2} y^{4}, x^{3} y^{2}\right)$ we have that $\check{I}^{2}=J \check{I}$ so that $f_{0}=\mu(\check{I})-1$.

Motivated by Theorem 4.1 and its proof, we can also show that the same sequence of ideals $I_{j}$, with $0 \leq j \leq d-1$, has special fiber rings $\mathcal{F}\left(I_{j}\right)$ with partially identical Hilbert polynomials to the one of $\mathcal{F}(I)$. Consistently with our previously established terminology, we denote with $f_{0}=f_{0}(I), f_{1}=f_{1}(I), \ldots, f_{d-1}=f_{d-1}(I)$ the coefficients of the Hilbert polynomial associated with the Hilbert function of the special fiber ring $\mathcal{F}(I)$.

Theorem 4.6. Let $(R, \mathfrak{m})$ be an analytically equidimensional local domain of dimension $d$ and let $I$ be an $\mathfrak{m}$-primary ideal. There exists a unique, largest ideal $I \subset I_{j} \subseteq \check{I}$, with $0 \leq j \leq d-1$ and the property that the corresponding Hilbert polynomial of the special fiber rings has coefficients satisfying

$$
f_{i}\left(I_{j}\right)=f_{i}(I), \quad i=0, \ldots, d-j-1 .
$$

In particular, the above result (see also the proof of Corollary 4.3) says that the degree one component $\check{I}$ of the $S_{2}$-ification of the Rees algebra $\mathcal{R}$ of $I$ can also be characterized as the largest ideal containing $I$ with the same multiplicity $f_{0}$. This statement is analogous to the characterization of the integral closure $\bar{I}$ of $I$ as the largest ideal containing $I$ with the same multiplicity $e_{0}$. However, our interest in $\check{I}$ has also been motivated by a recent connection, made in [18, with the computation of the core of $I$ (we recall that core $(I)$ is the intersection of all the reductions of $I$ ). The core of an ideal has lately been under much scrutiny: One of the classical motivations to study the core comes from the BriançonSkoda theorem, but more recently Hyry and Smith have shown that Kawamata's well-known conjecture on the existence of sections of line bundles is equivalent to a statement about the core of certain ideals in section rings. In a more general setting that ours, Polini, Ulrich 
and Vitulli have shown that $\operatorname{core}(I)=\operatorname{core}(\check{I})$. In view of our result, their statement can also be formulated by saying that two m-primary ideals $I \subset K$ with the same multiplicity $f_{0}$ also satisfy $\operatorname{core}(I)=\operatorname{core}(K)$.

\section{REFERENCES}

[1] S.S. Abhyankar, Local rings of high embedding dimension, Amer. J. Math. 89 (1967), 1073-1077.

[2] C. Ciuperca, First coefficient ideals and the $S_{2}$-ification of a Rees algebra, J. Algebra 242 (2001), 782-794.

[3] A. Corso and C. Polini, Links of prime ideals and their Rees algebras, J. Algebra 178 (1995), 224-238.

[4] A. Corso, L. Ghezzi, C. Polini and B. Ulrich, Cohen-Macaulayness of special fiber rings, Comm. Algebra (Kleiman's volume) 31 (2003), 3713-3734.

[5] S.D. Cutkosky, A new characterization of rational surfaces singularities, Invent. Math. 102 (1990) 157-177.

[6] D. Eisenbud, Commutative algebra with a view toward algebraic geometry, Springer-Verlag, New York, 1995.

[7] J. Elias and G. Valla, Rigid Hilbert functions, J. Pure and Appl. Algebra 71 (1991), 19-41.

[8] W. Heinzer, M.-K. Kim and B. Ulrich, The Gorenstein and complete intersection properties of associated graded rings, to appear in J. Pure and Appl. Algebra (Vasconcelos' volume).

[9] R. Hübl and C. Huneke, Fiber cones and the integral closure of ideals, Collect. Math. 52 (2001), 85-100.

[10] S. Huckaba and C. Huneke, Normal ideals in regular rings, J. reine angew. Math. 510 (1999), 63-82.

[11] C. Huneke, Hilbert functions and symbolic powers, Michigan Math. J. 34 (1987), 293-318.

[12] C. Huneke and J.D. Sally, Birational extensions in dimension two and integrally closed ideals, J. Algebra 115 (1988), 481-500.

[13] J. Lipman and B. Teissier, Pseudo-rational local rings and a theorem of Briançon-Skoda, Michigan Math. J. 28 (1981), 97-116.

[14] B. Mazur, Deformations of Galois representations and Hecke algebras, Harvard course notes, available by request from the author, 1994 .

[15] D.G. Northcott and D. and Rees, Reductions of ideals in local rings, Proc. Camb. Phil. Soc. 50 (1954), $145-158$.

[16] A. Ooishi, $\Delta$-genera and sectional genera of commutative rings, Hiroshima Math. J. 17 (1987), 361-372.

[17] C. Polini, B. Ulrich, W.V. Vasconcelos and R. Villarreal, Normalization of ideals, in preparation.

[18] C. Polini, B. Ulrich and M. Vitulli, Core of monomial ideals, in preparation.

[19] L.J. Ratliff and D.E. Rush, Two notes on reductions of ideals, Indiana Univ. Math. J. 27 (1978), 929-934.

[20] M.E. Rossi, A bound on the reduction number of a primary ideal, Proc. Amer. Math. Soc. 128 (2000), 1325-1332.

[21] J.D. Sally, Bounds for number of generators for Cohen-Macaulay ideals, Pacific J. Math. 63 (1976), 517-520.

[22] K. Shah, Coefficient ideals, Trans. Amer. Math. Soc. 327 (1991), 373-384.

[23] K. Shah, On the Cohen-Macaulayness of the fiber cone of an ideal, J. Algebra 143 (1991), 156-172.

[24] W.V. Vasconcelos, Hilbert functions, analytic spread and Koszul homology, Contemporary Mathematics 159 (1994), 410-422.

[25] W.V. Vasconcelos, The redution number of an algebra, Compositio Math. 104 (1996), 189-197.

[26] W.V. Vasconcelos, Cohomological degrees of graded modules, in Six Lectures on Commutative Algebra, J. Elias, J.M. Giral, R.M. Miró-Roig and S. Zarzuela (eds.), Progress in Mathematics 166, Birkhäuser, Basel, 1998, 345-392.

[27] W. V. Vasconcelos, The reduction numbers of an ideal, J. Algebra 216 (1999), 652-664.

[28] W.V. Vasconcelos, Multiplicities and reduction numbers, to appear in Compositio Math.

[29] M.T.R. Vaz Pinto, Structure of Sally modules and Hilbert functions, Ph.D. thesis, 1995.

[30] M.T.R. Vaz Pinto, Hilbert functions and Sally modules, J. Algebra 192 (1997), 504-523.

[31] A. Wiles, Modular elliptic curves and Fermat's last theorem, Ann. of Math. 141 (1995), 443-551. 
Department of Mathematics, University of Kentucky, Lexington, Kentucky 40506 - USA

E-mail address: corso@ms.uky.edu

Department of Mathematics, University of Notre Dame, Notre Dame, Indiana 46556 - USA

E-mail address: cpolini@nd.edu

Department of Mathematics, Rutgers University, New Brunswick, New Jersey 08901 - USA

E-mail address: vasconce@math.rutgers.edu 